\title{
Rumfitt on the logic of set theory
}

\author{
Øystein Linnebo
}

November 12, 2018

\begin{abstract}
According to a famous argument by Dummett, the concept of set is indefinitely extensible, and the logic appropriate for reasoning about the instances of any such concept is intuitionistic, not classical. But Dummett's argument is widely regarded as obscure. This note explains how the final chapter of Rumfitt's important new book advances our understanding of Dummett's argument but also points out some problems and unanswered questions. Finally, Rumfitt's reconstruction of Dummett's argument is contrasted with my own preferred alternative.
\end{abstract}

\section{Introduction}

What is the appropriate logic for use in set theory? This question has been controversial ever since the inception of Brouwer's intuitionism in the early twentieth century. Based on his radical anti-realist view that mathematics is concerned with mental constructions, Brouwer attacked both classical logic and transfinite set theory. But the subsequent mathematical development manifests a strong unwillingness to abandon "Cantor's paradise" of transfinite set theory. This prompts a refined question. Suppose we hold on to the essentials of transfinite set theory. Might there still be reason to doubt the appropriateness of classical logic for use in set theory?

Dummett famously thought so. We are not entitled to use classical first-order logic when reasoning about an "indefinitely extensible" domain such as the hierarchy of sets, he maintained. ${ }^{1}$ His argument is largely independent of his more familiar objections to classical logic based on his meaning-theoretic arguments and the version of anti-realism that these arguments were used to support. While this alternative Dummettian argument against the use of classical logic in set theory has intrigued and inspired many philosophers, ${ }^{2}$ it has never been developed in proper detail.

\footnotetext{
${ }^{1}$ As Rumfitt explains, (Dummett, 1993) provides a key reference, although the final chapter of (Dummett, 1991) is also important.

${ }^{2}$ See e.g. (Heck, Jr., 1993) and (Oliver, 1998).
} 
The final chapter of Rumfitt's important new book discusses the alternative Dummettian argument and some later developments, in particular by William Tait. ${ }^{3}$ In this note, I explain how Rumfitt's discussion advances our understanding of the argument but also point out some problems and unanswered questions. I begin by setting aside an aspect of Rumfitt's discussion that I shall not attempt to assess. Then, I present a schematic version of the Dummettian argument against the use of classical logic in set theory. After some remarks on the controversial notion of indefinite extensibility, I end by critically discussing Rumfitt's way of fleshing out the schematic argument and contrasting this way with my preferred alternative.

\section{Rumfitt's use of a negative translation}

As Rumfitt develops it, the challenge to the use of classical logic in set theory has two parts. First, it is argued that we cannot assume the Principle of Bivalence in set theory. That is, we are not entitled to assume that every sentence in the language of set theory is either true or false. Then, it is argued that the failure of Bivalence results in a failure of the Law of Excluded Middle and thus also of classical logic. While Rumfitt is broadly sympathetic with the first part of the argument (cf. p. 264), he wishes to resist its second part. He observes that the inference from the failure of bivalence to the failure of the Law of Excluded Middle needs to be defended. As he points out - and as Dummett would have been the first to agree - the tenability of the inference depends on the semantic theory that is appropriate for the language in question. In classical semantics, where "the meanings of the connectives are given by their truth-tables" (p. 264), the inference is straightforward. In a non-classical semantics, however, things are less clear cut.

Exploiting this leeway, Rumfitt tries to vindicate classical logic by defending a non-classical semantics for the language of set theory. He begins with a set-theoretic language $\mathcal{L}$ where bivalence fails. Then, he appeals to a non-classical semantics to argue that the set theoretic statements of working mathematicians should be translated into $\mathcal{L}$ in a non-homophonic way. Finally, he uses the logic of $\mathcal{L}$ and the non-homophonic translation to justify the use of classical logic in the language spoken by working mathematicians. The non-homophonic translation that Rumfitt favors is a version of the so-called "negative translation", which goes back to Gödel and Gentzen. It is a well known technical result that classical logic is vindicated when we first apply this translation and then reason intuitionistically.

While many theorists have invoked the negative translation, Rumfitt breaks new ground

\footnotetext{
${ }^{3}$ See (Rumfitt, 2015).
} 
by developing a detailed account of why this translation is appropriate, not merely an ad hoc trick. Indeed, his book can be seen as a sustained defense of the appropriateness of the translation. I shall not attempt to assess this defense but shall focus entirely on the first part of the challenge described above. That is, I shall explore why classical logic might be inappropriate in the original set theoretic language $\mathcal{L}$, while setting aside the question of how this language relates to the one spoken and written by working mathematicians.

\section{A schematic version of Dummett's argument}

Why should classical logic be impermissible in set theory? The problem Dummett has in mind concerns quantification.

For quantification to be defined, we must in some sense have determined or specified a domain. So how do we determine "the fundamental domains of quantification" that are studied in mathematics, such as the natural numbers or the real numbers? In empirical disciplines, it is relatively easy to determine a domain, Dummett thinks. It suffices to use a general term that is true of all and only the members of the domain. Assume we have a sharp definition of what it is to be a star, for example. Then we can rely on "external reality" to determine some objects that are all and only the stars (Dummett, 1993, p. 438). This reliance on "external reality" is not an option in mathematics, Dummett maintains. Although we know what it is to be a set, for example, there is no "external reality" on which we can rely to determine some objects that are all and only the sets. Since each fundamental mathematical domain is "supposed to be independent of how things happen to be in the world, [...] we must determine it" (ibid.). Mathematics is thus subject to the "further demand" that

we should 'grasp' the domain, that is, the totality of objects to which the term applies, in the sense of being able to circumscribe it by saying what objects, in general, it comprises - what natural numbers, or what real numbers, there are. (ibid.)

(The notion of "circumscribing" or determining some objects is not yet particularly clear. Some explications will be discussed in what follows.)

Suppose we are unable to satisfy this further demand by properly "circumscribing" the domain of all sets. Would this make us unable to quantify over all sets? While some commen- 
tators take this to be Dummett's view, ${ }^{4}$ a more careful reading shows this interpretation to be incorrect. Dummett accepts the possibility of quantification over an indefinitely extensible domain - provided that this quantification is intuitionistic, not classical.

There can be no objection to quantifying over all objects falling under some indefinitely extensible concept, say over everything we should, given an intelligible description of it, recognize as an ordinal number, provided that we do not think of the statements formed by means of such quantification as having determinate truth-conditions $[\ldots]$. They will not then satisfy the laws of classical logic, but only the weaker laws of intuitionistic logic. (Dummett, 1993, p. 440)

Where does this leave us? To make progress, I shall formulate a schematic version of Dummett's argument which I believe all of his readers can accept. I call the version "schematic" because it relies on a deliberately underspecified notion of determination. I shall later examine some candidate explications of this notion —-beginning with Rumfitt's own candidate - and assess the plausibility of the fleshed-out versions of the argument that result. Let "classical" and "intuitionistic quantification" be shorthand for quantification that is subject to the laws of classical or intuitionistic logic, respectively. My schematic reconstruction of Dummett's argument goes as follows.

(1) For classical quantification to be permissible, a domain must have been determined.

(2) The domain of set theory cannot be determined.

It follows that classical quantification is impermissible in set theory. Of course, this raises the question of what alternatives to classical quantification might be available in set theory. As we have seen, Dummett's answer takes the form of a third thesis:

(3) Intuitionistic quantification over the domain of set theory is permissible even though this domain cannot be determined.

\section{The "dark" notion of indefinite extensibility}

One of the aims of Rumfitt's reconstruction of the Dummettian argument is to "eschew the rather dark notion of 'indefinite extensibility'" (p. 264), which figures prominently in Dummett's own development of the argument and in later discussions of it. Here is one of Dummett's more useful explanations of the notion.

\footnotetext{
${ }^{4}$ See e.g. (Cartwright, 1994, p. 17) and (Boolos, 1993, pp. 215-16).
} 
A concept is indefinitely extensible if, for any definite characterization of it, there is a natural extension of this characterization, which yields a more inclusive concept; this extension will be made according to some general principle for generating such extensions, and, typically, the extended characterization will be formulated by reference to the previous, unextended, characterization (Dummett, 1963, pp. 195196)

The problem with this explanation should be obvious: it relies on an unexplained further notion of a "definite characterization". The difficulty should not be exaggerated, however: perhaps the further notion can be explained, even though Dummett failed to do so properly. In fact, relative to this further notion, Dummett's explanation of indefinite extensibility is tolerably clear. To see this, let $D(X)$ express that a concept $X$ admits of a "definite characterization", where this notion of definiteness has yet to be made precise. Then a concept $F$ is indefinitely extensible just in case there is an operation $\delta$ such that for any "definite" concept $X$ whose extension is contained in that of $F$, there is another $F$ which is not a member of $X$, namely $\delta(X)$. In symbols, a concept $F$ is indefinitely extensible just in case it satisfies the following condition:

$$
\forall X(D(X) \wedge X \subseteq F \rightarrow F(\delta(X)) \wedge \neg X(\delta(X)))
$$

where ' $X \subseteq F$ ' expresses that the extension of $X$ is contained in that of $F .^{5}$

What is the relation between a domain's being determined, which we discussed in the previous section, and the present notion of a concept's admitting of a definite characterization? Suppose the domain is to consist of all and only the things that fall under some concept. It seems reasonable to identify our two schematic notions by assuming that the domain is determined just in case we can provide a definite characterization of that concept. This conceptual identification allows us to kill two birds with one stone. The schematic argument from the previous section clearly requires an explication of the notion of a domain's being determined. Perhaps this explication can serve the additional purpose of defining the "further notion" on which Dummett's definition of indefinite extensibility depends, namely that of a concept's having a "definite characterization". If so, then Dummett's "dark notion" is

\footnotetext{
${ }^{5}$ When explicating indefinite extensibility, can we assume that sufficiently good sense can be made of the quantifier ' $\forall X$ '? After all, on some explications, indefinite extensibility is assimilated to the view that there is no maximally inclusive interpretation of the quantifiers. However, on the broadly Dummettian explication developed here, this assimilation is avoided. Even in the context of indefinite extensibility, there $i s$ a maximally inclusive interpretation of the quantifiers - albeit one that may only obey the laws of intuitionistic logic.
} 
ultimately no more obscure than a notion on which Rumfitt himself relies.

Of course, it is a different question whether the resulting notion of indefinite extensibility will do any explanatory work. To investigate this question, suppose that the concept of set is indefinitely extensible. That is, suppose:

$\left(2^{-}\right)$For any domain of sets that we can determine, we can use this determination to determine an even larger domain of sets.

Clearly, this claim entails premise (2): the domain of all sets cannot be determined if any determinable domain of sets can be extended to a larger such domain. Thus, one way to defend (2) is via a defense of the indefinite extensibility of the concept of set - in which case the notion of indefinite extensibility will certainly do explanatory work. We shall shortly see that on Rumfitt's development of the schematic argument, premise (2) is defended directly, meaning that he has indeed eschewed the notion of indefinite extensibility. By contrast, on the explication that I prefer, premise (2) is defended via the indefinite extensibility of the concept of set, as encapsulated in $\left(2^{-}\right)$.

\section{Determination as categorical characterizability}

How should we understand the notion of determination that figures in the schematic version of Dummett's argument? For the determination of mathematical domains to be problematicand thus for premise (2) to have any plausibility - the determination in question cannot be a trivial matter. It cannot suffice simply to state that the domain is to consist of all sets or of all objects whatsoever. I shall now consider Rumfitt's proposed explication of the notion of determination.

Finite mathematical domains are easily determined. Consider the domain of arithmetic modulo 12. This domain is exhaustively described by its addition and multiplication tables, which are easily surveyable. The determination of a mathematical domain is only problematic when the domain is infinite.

Rumfitt makes a distinctive and helpful proposal concerning the determination of an infinite domain. To "determine" a mathematical domain might be a matter of providing a categorical characterization of it. The paradigm example of this form of determination is Dedekind's celebrated characterization of the structure of the natural numbers in (Dedekind, 1888). Suppose we accept second-order logic. ${ }^{6}$ Let a simply infinite system be a collection $X$

\footnotetext{
${ }^{6}$ In fact, Rumfitt shows how one need not even go to full second-order logic to get this result, as so-called "ancestral logic" suffices.
} 
of objects, with a unique designated object $a$, and on which a dyadic relation $R$ is defined such that:

- $a$ is the unique initial object in the order $R$

- $R$ is one-to-one and functional

- The system is the smallest sequence that contains the initial object and is closed under $R$.

Then we can prove Dedekind's famous categoricity theorem, which states that any two simply infinite systems are isomorphic. As Rumfitt observes, this illustrates one way in which an infinite mathematical domain can be determined.

Let us plug Rumfitt's analysis of determination into our schematic argument. The result is the following fleshed-out argument:

$\left(1_{\mathrm{R}}\right)$ For classical quantification to be permissible, a domain must have been categorically characterized.

$\left(2_{R}\right)$ The domain of set theory cannot be categorically characterized.

These premises clearly imply that classical quantification over all sets is impermissible. So let us investigate whether the premises are defensible.

Let us begin with the second premise. Might the domain of sets too admit of a categorical characterization? Consider standard second-order Zermelo-Fraenkel set theory, $\mathrm{ZF}^{2}$. As Rumfitt reminds us, Ernst Zermelo proved a key result already in his justly famous (Zermelo, 1930): $\mathrm{ZF}^{2}$ is not categorical but quasi-categorical in the sense that, for any two models, one is an initial segment of the other. In fact, any strongly inaccessible rank $V_{\kappa}$ can be shown to provide a model of $\mathrm{ZF}^{2}$. While $\mathrm{ZF}^{2}$ — thanks to its reliance on second-order logicuniquely determines the "width" of the cumulative hierarchy of sets, the theory leaves open the "height" of the hierarchy. In other words, the theory ensures determinacy with respect to width but not with respect to height.

Might some extension of $\mathrm{ZF}^{2}$ ensure full categoricity by uniquely determining the height of its models as well as their width? One option is to add an axiom that specifies how many strongly inaccessible cardinals there are. We might for example add an axiom stating that there are no inaccessibles or that there are precisely $\omega$ many. Such a specification would fix the height of the models as the first inaccessible or the $\omega+1$ 'st, respectively. As Rumfitt 
observes, however, any axiom of this form would be restrictive in an unacceptable manner. For we can make good mathematical sense of their being more cardinals than we find in any of the resulting models. It would therefore be arbitrary and mathematically unmotivated to "truncate" the cumulative hierarchy in this way.

An alternative option is to pinpoint the height of the models by adding an axiom that requires the height to be maximal in some sense. This strategy is pursued by (McGee, 1997), who adds an axiom intended to ensure that the height of the models be as great as reality can accommodate. First, we tweak the theory $\mathrm{ZF}^{2}$ so as to allow urelemente; let $\mathrm{ZFU}^{2}$ be the result. Next, we let the quantifiers range over absolutely everything. Finally, we adopt an axiom stating that the urelemente form a set. McGee shows that the resulting theory is categorical.

Does this falsify Rumfitt's second premise, $\left(2_{R}\right)$ ? Like Rumfitt, I believe the answer is negative. But I am unconvinced by the reason he adduces, namely that the urelemente set axiom is false. My quarrel isn't with Rumfitt's claim that the "axiom" is false. He makes two observations intended to show that there are as many non-sets as there are sets, both of which strike me as convincing. Firstly, category theory requires as many categories as there are sets. Secondly, we might postulate as many primitive ordinals as there are sets. ${ }^{7}$ What I deny that is that the falsity of the urelemente set axiom gets to the heart of the matter. McGee's strategy can be seen to require nothing remotely as strong as the urelemente set axiom. All that the strategy requires is an axiom ensuring that the urelemente are not so numerous as to permit an even taller model of $\mathrm{ZFU}^{2}$. It suffices, for example, to require that there be no more urelemente than there are pure sets. This weaker requirement still entails categoricity yet is compatible with Rumfitt's examples of their being as many urelemente as pure sets. ${ }^{8}$

The problem with McGee's strategy isn't its appeal to the urelemente set axiom but its reliance on what Dummett calls "external reality". At the heart of the strategy is the idea of letting external reality determine the height of the models, thus turning Zermelo's quasicategoricity theorem into a full fledged categoricity result. There are precisely as many pure sets as there are objects in some independently given domain of absolutely everything. As Dummett insists, however, each fundamental mathematical domain is "supposed to be independent of how things happen to be in the world" (Dummett, 1993, p. 438). To let reality fix

\footnotetext{
${ }^{7}$ That is, ordinals not as the von Neumann ordinals of ZFC set theory but sui generis ordinals understood as order types of well orderings.

${ }^{8}$ The model that results from the addition of this particular axiom has the attractive feature that there is only one size of proper class, namely being equinumerous with the universe.
} 
the height of the cumulative hierarchy is to introduce an unacceptable arbitrariness - perhaps even contingency - into pure mathematics. Even if we could make sense of reality containing some determinate range of objects, we could use this range to make good mathematical sense of their being even more sets. ${ }^{9}$

Let me turn now to the first premise, $\left(1_{\mathrm{R}}\right)$, which says that classical quantification presupposes a domain that admits of a categorical characterization. Many philosophers would deny this, maintaining instead that classical quantification is available for domains that have only been determined in a far less demanding sense. ${ }^{10}$ Rumfitt's response would presumably be that these philosophers have failed to come to terms with the open-endedness of the hierarchy of sets. Indeed, he claims that "the semantic theories that best reflect the indeterminate height of the set-theoretic universe seem only to validate weaker than classical logics" (p. 285). Perhaps so. But the claim would need a more careful defense than it has received so far.

In fact, there is reason to doubt the claim, as can be seen by reflecting on some ideas due to Hilary Putnam. It is natural to wonder whether the arbitrariness that afflicts McGee's strategy can be eliminated by considering not only the largest model of $\mathrm{ZF}^{2}$ that reality in fact permits but all the possible models that reality might permit. (Putnam, 1967) makes a brilliant proposal along these lines, which is further developed in (Hellman, 1989). Consider the language of ordinary first-order set theory. We would like to provide a translation from this language into a second-order language that talks about possible models and their extensions. A simple example suffices to convey the idea, which is quite intuitive. Consider the claim that for every ordinal there is a greater ordinal: $\forall \alpha \exists \beta(\alpha<\beta)$. The so-called Putnam translation of this claim goes as follows: ${ }^{11}$

Necessarily, for every standard model and every object $\alpha$ that plays the role of an ordinal in this model, possibly there is an extended standard model containing an object $\beta$ that also plays the role of an ordinal, and according to which $\alpha$ is smaller than $\beta$.

We can now show that there are natural and plausible theories of ordinary first-order set theory and of possible models of set theory such that a sentence $\phi$ is a theorem of the former just in case its Putnam translation $\phi^{\mathrm{PT}}$ is a theorem of the latter. Sam Roberts has recently shown how this strategy can be used to validate Zermelo set theory with classical logic. ${ }^{12}$ However,

\footnotetext{
${ }^{9}$ See Section 6 below, as well as (Linnebo, 2010) for a more careful development.

${ }^{10}$ See e.g. (Rayo, 2003), (Williamson, 2003) and (Wright, 1998, Section 4).

${ }^{11}$ See (Hellman, 1989, pp. 73ff) for a general definition.

${ }^{12}$ Zermelo set theory is ZF minus the axiom scheme of Replacement. See (Roberts, 2014) for a precise statement of the result and proof.
} 
the modal theory employed in this strategy is plausibly taken to reflect "the indeterminate height of the set-theoretic universe" yet which validates classical logic.

\section{Determination as pure extensionality}

I shall end by outlining the explication of the notion of determination that I favor. I believe this explication is at least broadly in line with Dummett's intentions, and it gives rise to an interesting argument with considerable plausibility. The central idea is that a domain is determined just in case it admits of a purely extensional specification.

To unpack this idea, let me begin by observing that there are different ways to specify a collection. (I shall use the word "collection" in a deliberately unspecific way.) Some specifications are intensional, in the sense that they proceed by specifying a membership criterion. For example, since we know what it is for an object to be an ordinal, we can talk about the collection of all ordinals. Other specifications are extensional, in the sense that the collection is specified directly via its members, not by way of any membership criterion. A collection can for example be specified by providing a comprehensive list of its members.

The purest form of extensional specification is found when a collection is specified by means of the plurality - or the one or more objects - that are its members. To study this form of specification, I shall use the resources of plural logic, which makes the following additions to the expressive resources of ordinary singular logic:

- plural variables, typically written as $x x, y y$, etc.

- quantifiers binding such variables

- a logical predicate $\prec$, such that $x \prec y y$ means that $x$ is one of the objects $y y$

The idea is that a plural variable has one or more values. The semantic contribution of a plural variable is thus purely extensional. The variable contributes its one or more values, but no intension or membership criterion. I shall often refer to the semantic contribution of a plural variable as a plurality, even though this contribution should officially be characterized by means of plural locutions in our metalanguage. A plurality thus exhibits extensionality in its purest form: we are given one or more objects but no intension or membership criterion.

As mentioned, my central idea is that a domain is determined just in case it admits of a purely extensional specification. This can now be cashed out as admitting of specification 
by means of a plurality. Let us plug this analysis of "determination" into our schematic argument. We obtain the following fleshed-out argument:

$\left(1_{\mathrm{L}}\right)$ For classical quantification to be permissible, the domain must admit of specification by means of a plurality.

$\left(2_{\mathrm{L}}\right)$ The domain of set theory cannot be specified by means of a plurality.

$\left(3_{\mathrm{L}}\right)$ Intuitionistic quantification over the domain of set theory is permissible even though this domain cannot be specified by means of a plurality.

Clearly, the argument is valid: $\left(1_{\mathrm{L}}\right)$ and $\left(2_{\mathrm{L}}\right)$ entail that classical quantification is impermissible in set theory, while $\left(3_{\mathrm{L}}\right)$ ensures that intuitionistic quantification remains available. So again, we need to examine the plausibility of the premises.

Let us begin with $\left(2_{\mathrm{L}}\right)$. Since this has been an important theme in my earlier work, ${ }^{13} \mathrm{I}$ shall be brief. I have argued that, when a collection is specified in a purely extensional way, we can make good conceptual and mathematical sense of this collection defining a set. After all, we know exactly what the members of the would-be set are. And since the members are specified in a purely extensional way, no contradiction will ensue from positing or "forming" a set with precisely these members - if need be, outside of the pure extension with which we began. Since the target collection is specified in a purely extensional way, we have no problem conceiving of a richer situation in which this target collection defines a set. It would therefore be arbitrary and restrictive to deny the existence of the would-be set. I thus endorse:

$\left(2_{\mathrm{L}}^{-}\right)$For any domain of sets that can be specified by means of a plurality, we can use this specification to specify an even larger plurality of sets.

And as we have seen, this entails $\left(2_{\mathrm{L}}\right)$.

It is important to notice that the argument would be unconvincing if the members of the would-be set were specified in an intensional way. Assume for example that the desired set is specified by means of the membership criterion $x \notin x$. It is a simple truth of first-order logic - intuitionstic as well as classical - that there can be no set of the desired form. The desire is bound to be frustrated, in exactly the same way as the desire for a Russellian barber who shaves all and only those who don't shave themselves. Since both desires are logically

\footnotetext{
${ }^{13}$ See (Linnebo, 2010).
} 
incoherent, there is no mystery as to why they cannot be satisfied. ${ }^{14}$ We thus see that it is crucial how the collection of members of a would-be set is specified. Assume that the target collection is specified in a purely extensional way by means of a plurality. Then the target is modally rigid in a way that enables us to make sense of an ontologically richer situation where this very collection forms a set. Assume instead that the target collection is specified in an intensional way by means of a membership criterion. Then nothing is gained by considering a richer situation, since our non-rigid target will shift correspondingly.

I turn now to $\left(1_{\mathrm{L}}\right)$. This thesis is based on the idea that classical quantification rests on an instance-based conception of generality. Consider a true generalization $\forall x \phi(x)$ over a domain $D$. In virtue of what is this generalization true? On the classical conception of generality, the explanation of this truth invokes each and every instance of the form $\phi(a)$, for each object $a$ in $D .{ }^{15}$ However, this explanation presupposes that all of the instances are simultaneously available to serve in the explanans. And this, in turn, presupposes that $D$ admits of a purely extensional specification by means of a plurality. If the domain only admits of an intensional specification, there will be no stage of the process of considering ever richer ontologies at which all of its members are simultaneously available to figure in an explanation. This provides a good beginning of a defense of $\left(1_{\mathrm{L}}\right)$.

Assume that the domain of set theory cannot be specified in a purely extensional way by means of a plurality, as claimed by $\left(2_{\mathrm{L}}\right)$. Why should intuitionistic quantification remain permissible of this domain, as claimed by $\left(3_{\mathrm{L}}\right)$ ? Quantification over such a domain cannot be instance-based, since there is no stage at which all the instances are simultaneously available. So we need an alternative to the instance-based conception of generality. I believe an attractive alternative is provided by what we may call the generic conception of generality. Consider the following two generalizations.

(4) Every set has a singleton.

(5) No set is a member of the empty set.

To explain why these generalizations are true, there is no need to consider each and every

\footnotetext{
${ }^{14}$ To cite a truth of first-order logic should not be dismissed as merely to "wield the big stick, not to offer an explanation" (Dummett, 1991, p. 440). The non-existence of a Russellian barber requires no further explanation, and the set-theoretic analogue is no different. Like most others, Dummett failed to appreciate the importance of whether the desired set is specified in extensional or intensional manner.

${ }^{15}$ This idea figures in Dummett's writings, e.g. at (Dummett, 1981, p. 530): "We cannot take quantification over the totality of all objects as a sentence-forming operation which will always generate a sentence with a determinate truth-value; we cannot, in other words, interpret it classically as infinitary conjunction or disjunction." A closely related idea is also endorsed in central contributions to the burgeoning literature on metaphysical explanation or "grounding"; see e.g. (Fine, 2012, §7) and (Rosen, 2010, §8c).
} 
instance. Each generalization can be fully explained by citing completely general facts about the concepts of set, singleton, and empty set.

The generic conception of generality is explored and defended in some of my recent work, where I show that the conception licenses intuitionistic quantification but not classical. ${ }^{16}$ However, quantification restricted to any set is classical; for here, the instance-based conception is available. ${ }^{17}$ While my defense of these claims involves considerable technical work, the central idea is easily explained. On the generic conception, a universal generalization is very strong; in particular, it is stronger than the dual of the corresponding existential generalization. A universal generalization, understood generically, applies not only to the objects available at some particular stage of process of considering ever larger ontologies but also all objects that this process might ever introduce. By contrast, an existential generalization must, in order to be true at some particular stage of the process, have a witness that is available at this stage. Thus, while a universal generalization "looks ahead" at everything we might come to introduce into our domain, an existential generalization considers only what has already been introduced. This resulting non-duality of the two quantifiers gives rise to an intuitionistic logic, which establishes $\left(3_{\mathrm{L}}\right)$.

The arguments developed so far in this section are obviously not beyond reproach. The weakest part is probably the defense of $\left(1_{\mathrm{L}}\right)$, which relies on an alleged link between classical quantification and the instance-based conception of generality. But the link can be challenged. In particular, we observed in Section 5 that the Putnam-Hellman modal approach can be used to explain truths involving classical quantification. This observation suggests that such truths need not be understood in an instance-based way but might instead be grounded in appropriate modal facts. ${ }^{18}$

A promising response to this challenge is to develop a modal analogue of Dummett's demand that we "determine" a domain. Which are the modal facts on which the challenge to $\left(1_{\mathrm{L}}\right)$ relies? In particular, does the challenge rely on some "external modal reality"? If so, we might be able to envisage some form of "hypermodality" with respect to which there are even more possibilities. This raises the prospect that all of the individually but not jointly possible models of ZF2 that are exploited by the modal approach will nevertheless be jointly possible with respect to the "hypermodality". This would enable the process of specifying ever more

\footnotetext{
${ }^{16}$ See (Linnebo, 2016).

${ }^{17}$ This logic is known as semi-intuitionistic.

${ }^{18}$ Indeed, a version of this grounding of classical quantification in modal facts is developed and defended in my (Linnebo, 2013). I am currently undecided about whether this grounding is defensible or whether it succumbs to the response described in the next paragraph.
} 
inclusive interpretations of set theory to continue even further than what is deemed possible when relying on the original modality.

The generic conception of generality provides an attractive way to put this "revenge problem" to rest once and for all. When a generalization is explained not by its instances but by completely general facts about the concepts that are involved in the generalization, there can be no counter-instances to the generalization on some more inclusive interpretation of the quantifiers. For on any such interpretation, the mentioned conceptual facts will remain available and thus ensure that the generalization continues to hold. For example, even on a more inclusive interpretation of the quantifiers, the two generalizations (4) and (5) would remain true, and these truths would remain explained by completely general facts about the concepts involved in the generalizations.

Summing up, I have discussed two ways to flesh out the schematic version of Dummett's argument: Rumfitt's, which understands the key notion of determination as admitting of a categorical characterization; and my own, which understands this notion as admitting of a purely extensional specification. Although I have raised some problems with the former understanding, I wish to end on a conciliatory note. Rumfitt and I agree that the notion of determination has so far remained hopelessly obscure, and I commend him for proposing and developing an analysis of the notion which is finally clear enough to enable a proper evaluation. ${ }^{19}$

\section{References}

Benacerraf, P. and Putnam, H., editors (1983). Philosophy of Mathematics: Selected Readings, Cambridge. Cambridge University Press. Second edition.

Boolos, G. (1993). Whence the Contradiction? Proceedings of the Aristotelian Society, Suppl. Vol. 67:213-233. Reprinted in (Boolos, 1998).

Boolos, G. (1998). Logic, Logic, and Logic. Harvard University Press, Cambridge, MA.

Cartwright, R. L. (1994). Speaking of Everything. Noûs, 28:1-20.

Dedekind, R. (1888). Was Sind und Was Sollen die Zahlen? Vieweg, Braunschweig. English translation in (Ewald, 1996).

Dummett, M. (1963). The philosophical significance of Gödel's theorem. In Truth and Other Enigmas (1978), pages 186-214. Duckworth.

\footnotetext{
${ }^{19}$ Thanks to Salvatore Florio, Bob Hale, Jon Litland, and Agustín Rayo for discussions of the ideas developed in this note.
} 
Dummett, M. (1981). Frege: Philosophy of Language. Harvard University Press, Cambridge, MA, second edition.

Dummett, M. (1991). Frege: Philosophy of Mathematics. Harvard University Press, Cambridge, MA.

Dummett, M. (1993). What is mathematics about? In The Seas of Language, pages 429-445. Oxford University Press.

Ewald, W. (1996). From Kant to Hilbert: A Source Book in the Foundations of Mathematics, volume 2. Oxford University Press, Oxford.

Fine, K. (2012). Guide to ground. In Correia, F. and Schnieder, B., editors, Metaphysical Grounding, pages 37-80. Cambridge University Press.

Heck, Jr., R. G. (1993). Critical notice of Michael Dummett, Frege: Philosophy of mathematics. Philosophical Quarterly, 43:223-33.

Hellman, G. (1989). Mathematics without Numbers. Clarendon, Oxford.

Linnebo, Ø. (2010). Pluralities and sets. Journal of Philosophy, 107(3):144-164.

Linnebo, Ø. (2013). The potential hierarchy of sets. Review of Symbolic Logic, 6(2):205-228.

Linnebo, Ø. (2016). Generalizations explained: A truth-maker semantics. Unpublished MS.

McGee, V. (1997). How We Learn Mathematical Language. Philosophical Review, 106(1):3568.

Oliver, A. (1998). Hazy totalities and indefinitely extensible concepts. Grazer Philosophische Studien, 55:25-50.

Putnam, H. (1967). Mathematics without Foundations. Journal of Philosophy, LXIV(1):5-22. Reprinted in (Benacerraf and Putnam, 1983) and (Putnam, 1975).

Putnam, H. (1975). Mathematics, Matter and Method. Cambridge University Press, Cambridge.

Rayo, A. (2003). When does 'everything' mean everything? Analysis, 63(278):100-106.

Roberts, S. (2014). Reflection in the modal structural setting. Under review.

Rosen, G. (2010). Metaphysical dependence: Grounding and reduction. In Hale, B. and Hoffmann, A., editors, Modality: Metaphysics, Logic, and Epistemology, pages 109-36. Oxford University Press.

Rumfitt, I. (2015). The Boundary Stones of Thought: An Essay in the Philosophy of Logic. Oxford University Press.

Williamson, T. (2003). Everything. In Hawthorne, J. and Zimmerman, D., editors, Philosophical Perspectives 17: Language and Philosophical Linguistics. Blackwell, Boston and Oxford. 
Wright, C. (1998). The Harmless Impredicativity of $N^{=}$(Hume's Principle). In Schirn, M., editor, Philosophy of Mathematics Today. Clarendon, Oxford.

Zermelo, E. (1930). Über Grenzzahlen und Mengenbereiche. Fundamenta Mathematicae, 16:29-47. Translated in (Ewald, 1996). 